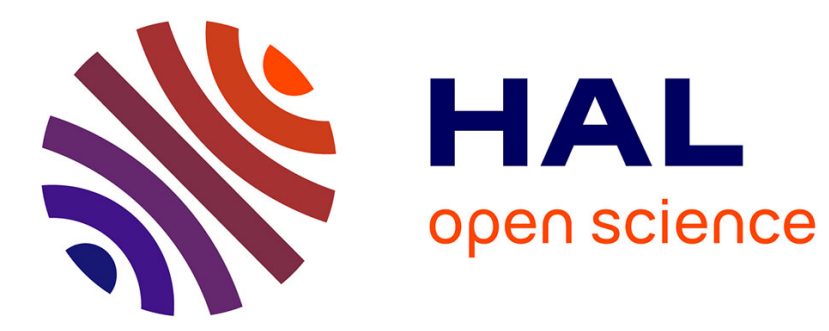

\title{
More on square-free words obtained from prefixes by permutations \\ Pascal Ochem
}

\section{To cite this version:}

Pascal Ochem. More on square-free words obtained from prefixes by permutations. Fundamenta Informaticae, 2014, Russian-Finnish Symposium in Discrete Mathematics, 132 (1), pp.109-112. 10.3233/FI-2014-1035 . lirmm-01375793

\section{HAL Id: lirmm-01375793 https://hal-lirmm.ccsd.cnrs.fr/lirmm-01375793}

Submitted on 3 Oct 2016

HAL is a multi-disciplinary open access archive for the deposit and dissemination of scientific research documents, whether they are published or not. The documents may come from teaching and research institutions in France or abroad, or from public or private research centers.
L'archive ouverte pluridisciplinaire HAL, est destinée au dépôt et à la diffusion de documents scientifiques de niveau recherche, publiés ou non, émanant des établissements d'enseignement et de recherche français ou étrangers, des laboratoires publics ou privés. 


\title{
More on square-free words obtained from prefixes by permutations
}

\author{
Pascal Ochem \\ LIRMM, CNRS, Univ. Montpellier 2 \\ ochem@lirmm.fr
}

May 23, 2013

\begin{abstract}
An infinite square-free word $w$ over the alphabet $\Sigma_{3}=\{0,1,2\}$ is said to have a $k$-stem $\sigma$ if $|\sigma|=k$ and $w=\sigma w_{1} w_{2} \ldots$ where for each $i$, there exists a permutation $\pi_{i}$ of $\Sigma_{3}$ which extended to a morphism gives $w_{i}=\pi_{i}(\sigma)$. Harju proved that there exists an infinite $k$-stem word for $k=1,2,3,9$ and $13 \leq k \leq 19$, but not for $4 \leq k \leq 8$ and $10 \leq k \leq$ 12. He asked whether $k$-stem words exist for each $k \geq 20$. We give a positive answer to this question. Currie has found another construction that answers Harju's question.
\end{abstract}

\section{Introduction}

An infinite square-free word $w$ over the alphabet $\Sigma_{3}=\{0,1,2\}$ is said to have a $k$-stem $\sigma$ if $|\sigma|=k$ and $w=\sigma w_{1} w_{2} \cdots$ where for each $i$, there exists a permutation $\pi_{i}$ of $\Sigma_{3}$ which extended to a morphism gives $w_{i}=\pi_{i}(\sigma)$. Harju [3] proved that there exists an infinite $k$-stem word for $k=1,2,3,9$ and $13 \leq k \leq$ 19 , but not for $4 \leq k \leq 8$ and $10 \leq k \leq 12$ and asked whether $k$-stem words exist for each $k \geq 20$. We construct $k$-stem words for each $20 \leq k \leq 10000$ in Section 3 and for every $k \geq 23$ in Section 4. Currie [2] has found another construction that answers Harju's question.

Let $t=012021012102012021020121012 \ldots$ denote the fixed point of the morphism $0 \mapsto 012,1 \mapsto 02,2 \mapsto 1$. By definition, $t$ contains neither 010 nor 212 as a factor. Harju [3] also asked whether $t$ has a $k$-stem factorization for some $k \geq 3$. We give a negative answer in Section 2. This result has also been obtained by Harju and Müller [4].

\section{$2 k$-stem factorization of $t$}

Theorem 1 No suffix of $t$ admits a $k$-stem factorization for any $k \geq 3$. 
Proof. By previous results [3], we only need to consider the cases $k=9$ and $k \geq 13$.

A computer check shows that no factor $f$ of $t$ of length 18 is such that the suffix of length 9 of $f$ is a permutation of the prefix of length 9 of $f$. This rules out the case $k=9$.

A computer check shows that every factor $f$ of $t$ of length 12 contains a factor $a 0 a$ with $a \in \Sigma_{3}$. By symmetry, it also contains a factor $b 2 b$ with $b \in \Sigma_{3}$. Remember that 010 and 212 are not factors of $t$. A permutation of $\Sigma_{3}$ mapping 0 to 1 (resp. mapping 2 to 1 ) cannot be applied to $f$, since it would produce a factor $c 1 c$ with $c \in \Sigma_{3}$ that cannot appear in $t$. There remain two possible permutations, namely the identity and the permutation swapping 0 and 2 , but an infinite square free word cannot be obtained by a concatenation of only two distinct factors. This rules out the case $k \geq 13$.

\section{$3 k$-stem words for $20 \leq k \leq 10000$}

Theorem 2 There exist $k$-stem words for every $20 \leq k \leq 10000$.

Proof. Let $\pi$ be the permutation (012). We say that a morphism $h: \Sigma_{3}^{*} \rightarrow \Sigma_{3}^{*}$ is circular if $h(1)=\pi(h(0))$ and $h(2)=\pi(h(1))$. For every $20 \leq k \leq 10000$, we found a word $w_{k}$ such that $\left|w_{k}\right|=c_{k} \times k$ and the circular morphism $m$ defined by $m(0)=w_{k}$ is square-free. We have $c_{k}=8$ for $20 \leq k \leq 22$ and $c_{k}=1$ for $23 \leq k \leq 10000$. Square-freeness is checked using the result of Crochemore [1] that a uniform morphism $h$ is square-free if and only if the $h$-images of squarefree words of length 3 are square-free. Since we consider circular morphisms, we only need to check the images of 010 and 012 .

These are our words $w_{k}$ for $20 \leq k \leq 22$, where $\left|w_{k}\right|=8 k$.

$$
\begin{aligned}
w_{20}= & 012102010210121021201020121012010201202120102120210201021012 \\
& 021201020120212012101202101210212021020121012021201210120102 \\
& 1202101210212021020102120102012021201210 \\
w_{21}= & 012021020102120102012021012010201210201021201210212021012021201 \\
& 021012010201210201021012021020102120102012102120121012021012102 \\
& 120102101210201210120210201202120102120210 \\
w_{22}= & 012021020102120210201202101201020121012010212012102120210121021201 \\
& 021012010201210120102101202102010212021020121021201210120212012102 \\
& 12010210121020102101202102012021201020120210
\end{aligned}
$$

Consider now the case $k \geq 23$, where $\left|w_{k}\right|=k$. Let $t^{\prime}=012021020121012 \ldots$ denote the infinite suffix of $t$ obtained from $t$ by deleting the first 12 letters. To speed up the search of a suitable $w_{k}$, we impose that $w_{k}=\operatorname{pr} 120210$ where $p$ is the prefix of length $k-22$ of $t^{\prime}$ and $r$ belongs to the set $S$ of size 13 below, except that $r=2102010210121020$ for $k=26$.

$$
S=\{0102120121020102,0120102120121020,0212012101201020,
$$
1012010212012102, 1021201021012102, 1201020121020102, 1202120121020102, 
$1210120212012102,1210201202120102,1210201210120102,2010210121020102$, $2102120121020102,2120102101201020\}$

\section{$4 \quad k$-stem words for large $k$}

Theorem 3 There exist $k$-stem words for every $k \geq 1$ except for $4 \leq k \leq 8$ and $10 \leq k \leq 12$

Proof. Consider the following morphism $d$, having two possible images for each letter: one image of length 17 and one image of length 18.

$$
\begin{aligned}
0 & \mapsto\left\{\begin{array}{l}
01202120102120210 \\
012021020102120210
\end{array}\right. \\
1 & \mapsto\left\{\begin{array}{l}
12010201210201021 \\
120102101210201021
\end{array}\right. \\
2 & \mapsto\left\{\begin{array}{l}
20121012021012102 \\
201210212021012102
\end{array}\right.
\end{aligned}
$$

Again, using the result of Crochemore [1], $d$ is shown to be square-free by checking that the $d$-images of square-free words of length $\max \left(3,\left[\frac{18-3}{17}\right]\right)=3$ are square-free. Since the restriction of $d$ to images of length 17 (resp. 18) is circular, we only need to check the images of 010 and 012 are square-free. For each of the factors 010 and 012 , we actually have $2^{3}$ images to check since each of the letters can be mapped either to its image of length 17 or 18 .

If $m$ is a square-free circular morphism, then for every $d$-image $w_{0}$ of $m(0)$, the circular morphism defined by $0 \mapsto w_{0}$ is square-free. This means that given a $k$-uniform square-free circular morphism, we can construct a $k^{\prime}$-uniform square-free circular morphism for every $k^{\prime}$ such that $17 k \leq k^{\prime} \leq 18 k$.

Now we prove that there exist $k$-uniform square-free circular morphisms for every $k \geq 23$. We start with the cases $k \in[23,10000]$ which are proved in the previous section. They imply the cases $k \in \bigcup_{23 \leq p \leq 10000}[17 p, 18 p]$, i.e., $k \in[391,180000]$. We then obtain every $k \geq 23$ by induction.

\section{Concluding remarks}

We have proved that there exist infinite square-free ternary words with a $k$-stem factorization for every $k$ except $4 \leq k \leq 8$ and $10 \leq k \leq 12$. We conjecture that there exist $k$-stem words of the form $w_{k}=p r 120210$ described in the proof of Theorem 2 for every $k \geq 23$, rather than $23 \leq k \leq 10000$. Before we found the morphism of the proof of Theorem 3, we pushed the verification to up to 10000 in order to find a way to prove this conjecture, but the proof of Theorem 3 only requires a verification for $23 \leq k \leq 390$.

From the proof of Theorem 3 , we see that the number of $k$-uniform squarefree circular morphisms is exponential in $k$, at least about $\left(\begin{array}{c}2 k / 35 \\ k / 35\end{array}\right) \approx 2^{2 k / 35}$. We conjecture the following: 
Conjecture 4 The growth rate of ternary words defining a square-free circular morphism exists and is equal to the growth rate $1.3017 \ldots$ of ternary square-free words.

See Shur [5] for more information on the growth rate of ternary square-free words.

\section{References}

[1] M. Crochemore. Sharp characterizations of squarefree morphisms, Theoret. Comput. Sci. 18(2) (1982), 221-226.

[2] J. Currie. Infinite ternary square-free words concatenated from permutations of a single word, Theoret. Comput. Sci. 482 (2013), 1-8.

[3] T. Harju. Square-free words obtained from prefixes by permutations, Theoret. Comput. Sci. 429 (2012), 128-133.

[4] T. Harju and M. Müller. Square-free words generated by applying permutations to a prefix, in Proceedings of the Second Russian Finnish Symposium on Discrete Mathematics, RuFiDim II, (V. Halava, J. Karhumäki, Y. Matiyasevich, eds.) (2012), 86-91.

[5] A. Shur. Growth rates of complexity of power-free languages, Theoret. Comput. Sci. 411(34-36) (2010), 3209-3223. 